\title{
Mutations of epidermal growth factor receptor in colon cancer indicate susceptibility or resistance to gefitinib
}

\author{
XIANG ZHANG $^{1,2}$, HISASHI NAGAHARA ${ }^{1,2}$, KOSHI MIMORI $^{1}$, HIROSHI INOUE ${ }^{1}$, \\ TETSUJI SAWADA ${ }^{2}$, MASAICHI OHIRA ${ }^{2}$, KOSEI HIRAKAWA ${ }^{2}$ and MASAKI MORI ${ }^{1}$ \\ ${ }^{1}$ Department of Surgery and Molecular Oncology, Medical Institute of Bioregulation, Kyushu University, \\ 4546 Tsurumihara, Beppu 874-0838; ${ }^{2}$ Department of Surgical Oncology, Osaka City University \\ Graduate School of Medicine, 1-4-3 Asahi-machi, Abeno-ku, Osaka 545-8585, Japan
}

Received October 9, 2007; Accepted December 3, 2007

\begin{abstract}
Somatic mutations of the epidermal growth factor receptor $(E G F R)$ gene may predict the sensitivity of nonsmall cell lung cancers to gefitinib. In our previous study, we identified somatic mutations in the tyrosine kinase domain of the EGFR gene in $12.1 \%$ of colon cancer cases. Herein, we focus on whether the mutations are associated with the sensitivity of colon cancer to gefitinib. The E749K mutation in exon 19 and E762G and A767T mutations in exon 20 were introduced into the full-length EGFR coding sequence in a pBKCMV-hEGFR vector by site-directed mutagenesis and transfected into LS174T cells. The sensitivity to gefitinib was compared between the transfected LS174T and the parental cells by a cytotoxic assay. The LS174T cells with E749K were significantly $(\mathrm{p}<0.05)$ more responsive to gefitinib than the parental cells. On the other hand, LS174T cells with E762G or A767T were significantly $(\mathrm{p}<0.05)$ more resistant than the parental cells. In conclusion, detection of somatic mutations in the epidermal growth factor receptor may play an important role in predicting sensitivity to gefitinib in colon cancer.
\end{abstract}

\section{Introduction}

Epidermal growth factor receptor (EGFR) is a transmembrane glycoprotein (1). Binding of ligands EGF, HGF or TGF $\alpha$ to the extracellular domain of EGFR induces either homodimerization or heterodimerization with other closely related receptors such as HER2/neu. Dimerization enables ATP binding at the ATP cleft within the tyrosine kinase (TK) domain of EGFR

Correspondence to: Professor Masaki Mori, Department of Surgery and Molecular Oncology, Medical Institute of Bioregulation, Kyushu University, 4546 Tsurumihara, Beppu 874-0838, Japan

E-mail: mmori@tsurumi.beppu.kyushu-u.ac.jp

Key words: colon cancer, epidermal growth factor receptor, gefitinib, somatic mutation and phosphorylation of tyrosine residues within the intracellular domain of EGFR. These phosphorylated residues serve as docking sites for a range of proteins, the recruitment of which leads to the activation of intracellular signaling pathways (2).

Aberrant activation of EGFR abnormally activates downstream signaling pathways and plays an important role in cancer cell development and progression in various tumors of epithelial origin, including cancers of the lung, breast, head and neck, and bladder $(3,4)$. Two classes of drugs have been approved for the treatment of these cancers. One class is the anti-EGFR-blocking monoclonal antibodies (MAbs), such as cetuximab, which prevents ligand-EGFR binding. The other class is the selective EGFR small molecule tyrosine kinase inhibitors (TKIs), such as gefitinib and erlotinib, which prevent ATP-EGFR binding. Cetuximab has been approved for the treatment of colorectal cancer in the US and gefitinib and erlotinib for lung cancer (5-8).

In clinical studies, the response rate of gefitinib has been shown to vary among races. For example, $27.5 \%$ of Japanese patients with non-small cell lung cancers (NSCLC) exhibited a response to gefitinib, compared to $10.4 \%$ of European patients (9). According to an epidemiological study in the US, partial clinical response to gefitinib has been observed most frequently in women, in non-smokers, and in patients with adenocarcinoma (10-12). Therefore, much research has focused on selection of the best indicator to predict patients who would benefit from gefitinib treatment.

Lynch et al and Paez et al reported that mutations in exons 18-21 coding the EGFR kinase domain were related to a clinical response to TKIs in NSCLC (13-15). In a previous study, we identified somatic mutations in the TK domain of the EGFR gene in 4 of $33(12.1 \%)$ cases of colorectal cancer (CRC). Two were the substitution E749K in exon 19, one was E762G in exon 20 and one was A767T in exon 20 of the EGFR gene (16). However, the relationship between mutations in EGFR and susceptibility to gefitinib in CRC has not been elucidated. In the current study, we compared the sensitivity or resistance to gefitinib among CRC cell lines of wild-type and the three mutated EGFR constructs (E749K, E762G and A767T) to determine whether these mutations could be useful predictors of gefitinib response. 


\section{Materials and methods}

Expression constructs. We prepared the EGFR vector pLSX (provided by Professor A. Takayanagi, Keio University, Tokyo, Japan). Subsequent to cutting 3.9-kb EGFR at the $X h o$ site from the pLSX vector, EGFR was inserted into the pBKCMV-hEGFR vector (Stratagene, La Jolla, CA, USA) (17). In addition to the wild-type plasmid of EGFR, we established mutant clones that had been discovered in human cases of sporadic colorectal cancer in our previous study (16). We artificially altered nucleotides at $2245 \mathrm{G}>\mathrm{A}$ (E749K) in exon 19 and $2285 \mathrm{~A}>\mathrm{G}(\mathrm{E} 762 \mathrm{G})$ and $2299 \mathrm{G}>\mathrm{A}$ (A767T) in exon 20. To establish these mutant clones, we followed the manufacturer's protocol of the QuickChange site-directed mutagenesis kit (Stratagene) with the following oligonucleotide primers: 5'-cgctatcaaggaattaagaaaagcaacatctccgaaagc-3' and 5'-gctttcggagatgttgcttttcttaattcettgatagcg-3' for E749K; 5'-caa ggaaatcctcgatggagctacgtgatggc-3' and 5'-gccatcacgtaggct ccatcgaggatttccttg-3' for E762G; 5'-agcctacgtgatgaccagcgtgg acaacc-3' and 5'-ggttgtccacgctggt catcacgtaggct-3' for A767T. All constructs were fully sequenced (18).

Transfection. Cell lines derived from human colorectal cancer LS174T were obtained from the Cell Resource Center for Biomedical Research Institute of Development, Aging and Cancer (Tohoku University, Sendai, Japan). For transfection, $1 \times 10^{5}$ LS174T cells were seeded in DMEM (Invitrogen, Carlsbad, CA, USA) containing 10\% FBS on 6-well culture plates. After $24 \mathrm{~h}$, medium was exchanged with $2 \mathrm{ml}$ DMEM supplemented with $0.2 \%$ FCS and cultured for an additional $4 \mathrm{~h}$. Transfection was performed using Fugene 6 transfection regent (Roche Molecular Biochemicals, Indianapolis, IN, USA), according to the manufacturer's instructions, with minor modifications. Briefly, $1 \mu \mathrm{g}$ plasmid DNA purified by an endotoxin-free purification system (Qiagen, Tokyo, Japan) was diluted in $50 \mu 1$ OPTI-MEM1 (Invitrogen). The diluted DNA was mixed with the $3 \mu \mathrm{l}$ reagent, which was prediluted in $50 \mu \mathrm{l}$ OPTI-MEM1. The mixture of DNA and reagent was added to the culture medium in a drop-wise manner.

Western blot analysis. Cells were washed with PBS. For detection of phosphorylated EGFR protein, cells were lysed for 20 min in RIPA buffer containing sodium orthovanadate (Calbiochem, San Diego, CA, USA) and Complete protease inhibitor cocktail (Roche) on ice. For detection of total EGFR protein, cells were lysed for $20 \mathrm{~min}$ in RIPA buffer containing Complete protease inhibitor cocktail on ice. The lysate was homogenized by passing the sample through a 21-gauge needle. In brief, for immunoblot analysis, the samples were subjected to SDS-PAGE on $15 \%$ acrylamide gels under reducing conditions and transferred to Immobilon-P membranes (Millipore, Bedford, MA, USA). After blocking with $5 \%$ non-fat dry milk and $0.05 \%$ Tween-20 in PBS, blots were incubated with EGFR antibody (1:2500, BD Biosciences, San Jose, CA, USA) and/or phosphorylated EGFR antibody (1:1000, BD Biosciences). After several washing steps, blots were incubated for $1 \mathrm{~h}$ with goat anti-mouse IgG (1:5000, Dako Japan, Kyoto, Japan) coupled to horseradish peroxidase then washed extensively. Enhanced chemiluminescence detection reagents (Amersham Biosciences, Piscataway, NJ,

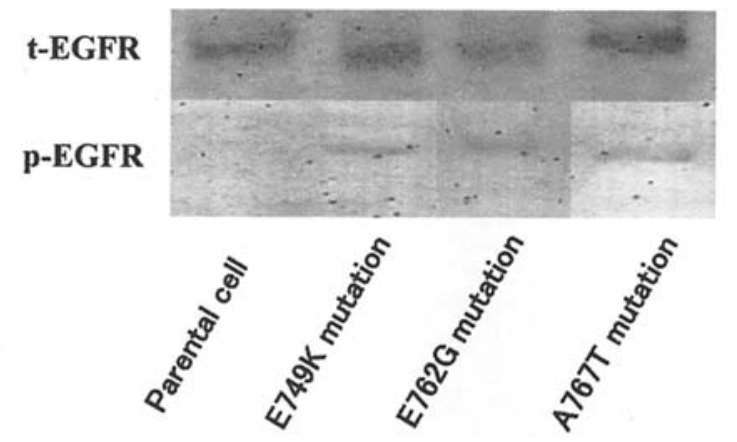

Figure 1. We measured the levels of total or phosphorylated EGFR protein by using EGFR or phosphorylated EGFR antibody. The upper lane shows that the transfectants and the parental cells have equivalent expression levels of total EGFR protein. The lower lane shows that the transfectants have a higher expression level of the phosphorylated EGFR protein compared to parental cells.

USA) were applied to detect the signal of the antigen-antibody reaction.

Cytotoxic assay. Proliferation was determined using 3-(4,5dimethylthiazol-2-yl)-2, 5-diphenyl tetrazolium bromide (MTT) assay (Roche). Logarithmically growing cells were seeded at $8.0 \times 10^{3}$ and $1.0 \times 10^{4}$ cells/well in microtiter plate wells (96 wells, flat bottom) in a final volume of $100 \mu \mathrm{l}$ DMEM with $10 \%$ FBS per well under a humidified atmosphere $\left(37^{\circ} \mathrm{C}\right.$ and $\left.5 \% \mathrm{CO}_{2}\right)$. After 0 and $72 \mathrm{~h}$ of incubation, $10 \mu \mathrm{l}$ of MTT labeling reagent (final concentration $0.5 \mathrm{mg} / \mathrm{ml}$ ) was added to each well. The microtiter plate was incubated for $4 \mathrm{~h}$ in a humidified atmosphere, then solubilization solution $(100 \mu 1)$ was added to each well. The plate was allowed to stand overnight in the incubator under a humidified atmosphere. After checking for complete solubilization of the purple formazan crystals, the spectrophotometrical absorbance of the samples was measured using a model 550 microplate reader (Bio-Rad Laboratories, CA, USA) at a wavelength of $570 \mathrm{~nm}$ corrected to $655 \mathrm{~nm}$. Cells were exposed to varying concentrations $(0,0.1,1,10,20,30,50,80 \mu \mathrm{M})$ of gefitinib. All studies were performed with six replicates.

\section{Results}

The missense mutations E749K in exon 19, E762G and A767T in exon 20 were introduced into the full-length EGFR coding sequence in a $\mathrm{pBKCMV-hEGFR} \mathrm{vector} \mathrm{by} \mathrm{the} \mathrm{use} \mathrm{of}$ a site-directed mutagenesis system. We performed immunoblotting of extracts from the parental and transfected LS174T cells expressing the various mutated EGFR to assess various aspects of protein activity. We measured the expression level of total EGFR protein using an EGFR antibody. The upper lane in Fig. 1 shows that the transfectants and the parental cells have equivalent expression levels of total EGFR protein, indicating that the mutations do not affect the stability of the protein. We measured the expression levels of activated EGFR protein by using a phosphorylated EGFR antibody. The lower lane in Fig. 1 shows the transfectants have higher expression levels of the phosphorylated EGFR protein than the parental cells, indicating that the transfectants have a greater ability to phosphorylate EGFR than the parental cells. Consistent with 


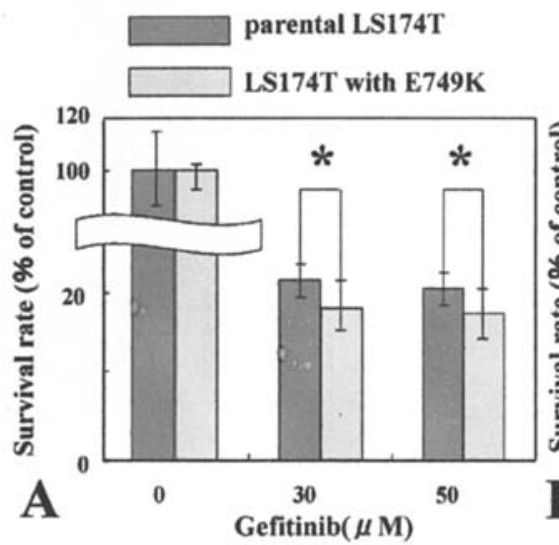

Gefitinib $(\mu \mathrm{M})$
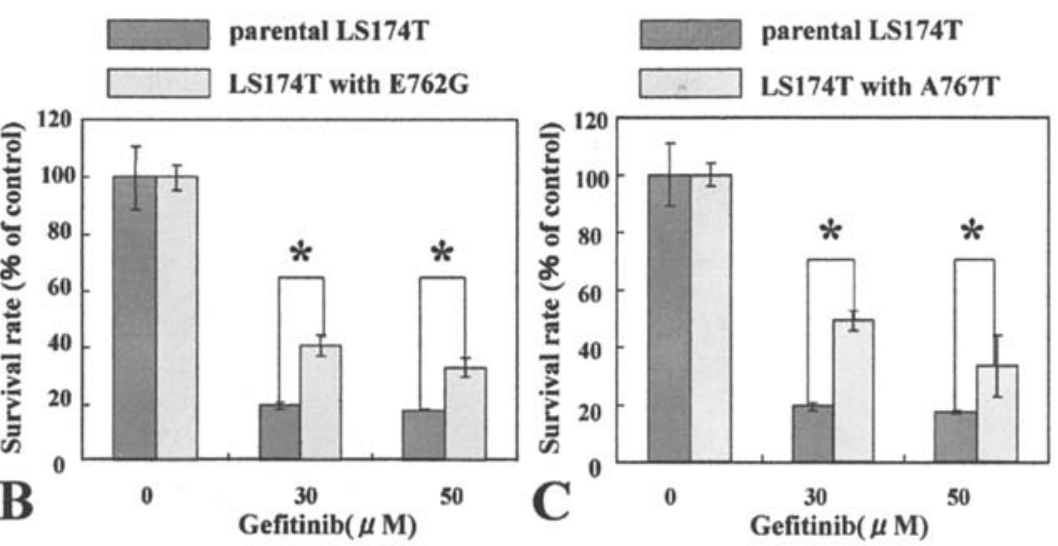

$* P<0.005$

Figure 2. Sensitivity to gefitinib differs between the transfected and the parental LS174T cells. (A) The cells with E749K (gray bar) are more sensitive than the parental cells (black bar) at the concentrations of gefitinib, 30 and $50 \mu \mathrm{M}$. The cells with E762G (gray bar, B) and the cells with A767T (gray bar, C) are more resistant than the parental cell (black bar, B and C) at the concentrations of gefitinib, 30 and $50 \mu \mathrm{M}$.

this result, these EGFR mutants induced markedly high levels of tyrosine-autophosphorylation compared to wild-type EGFR.

We compared the sensitivity to gefitinib between the transfected and the parental LS174T cells using a cytotoxic assay. The LS174T cells with E749K mutant constructs were more sensitive than the parental cells to gefitinib concentrations of $10 \mu \mathrm{M}$ ( $<<0.05), 20 \mu \mathrm{M}$ ( $<<0.05)$ (data not shown), $30 \mu \mathrm{M}$ $(\mathrm{p}<0.005)$ and $50 \mu \mathrm{M}(\mathrm{p}<0.005)($ Fig. 2A). The LS174T cells with E762G or A767T mutant constructs were more resistant than the parental cells to gefitinib concentrations of $10 \mu \mathrm{M}$ (p<0.05), $20 \mu \mathrm{M}(\mathrm{p}<0.05)$ (data not shown), $30 \mu \mathrm{M}(\mathrm{p}<0.005)$ and $50 \mu \mathrm{M}(\mathrm{p}<0.005)$ (Fig. 2B and C).

\section{Discussion}

In recent studies, $\sim 90 \%$ of mutations resulting in an improved clinical response to TKIs in NSCLC were identified as either an in-frame deletion 747-750 in exon 19 or missense mutation L858R in exon 21 (12). However, missense mutation T790 M and NPG insertion mutation 770-771 in exon 20 have been reported to decrease the clinical response to TKIs in NSCLC $(19,20)$.

The formation of homo- or hetero-dimer is essential for the activation of EGFR and is mediated by ligand-EGFR binding in the wild-types (2). Lynch et al (13) and Greulich et al (21) reported that a conformational alteration induced by in-frame deletion 747-750 in exon 19 or missense mutation L858R in exon 21 stabilized the dimer without ligands. This facilitates abnormal ATP or TKI-EGFR binding, resulting in either aberrant activation of the EGFR pathway or increasing sensitivity to TKIs.

In our Western blot analysis in the absence of ligands, transfected LS174T cells expressing various mutated EGFR showed phosphorylated EGFR whereas the parental wild-type cells did not show any phosphorylation. This result suggests that the E749K, E762G and A767T mutations induce a conformational alteration and stabilize the dimer, resulting in ligand-independent activation of EGFR. Thus, we predict that colorectal cancers with these mutations are resistant to cetuximab, which exerts its anti-tumor effect by inhibiting
ligand-EGFR binding. Therefore, gefitinib would be more effective than cetuximab in colorectal cancer for E749K, which also showed increased sensitivity to gefitinib in our cytotoxic assay. On the other hand, E762G and A762T which exist in exon 20, decreased sensitivity to gefitinib, similarly to the missense mutation $\mathrm{T} 790 \mathrm{M}$ or NPG insertion mutation D770-771. Kobayashi et al and Greulich et al reported that the irreversible TKI, CL-387,785, showed an anti-proliferative effect on NSCLC cells with the missense mutation T790 M or the NPG insertion mutation D770-771 $(21,22)$. Thus, we predict that CL-387,785 may be more effective than cetuximab or gefitinib in colorectal cancers with E762G and A767T EGFR mutations.

Gefitinib has not been approved for colorectal cancer treatment. However, 4 of 33 Japanese colorectal cancer cases showed EGFR mutations in our previous study, and this study clearly demonstrates that two cases with the E749K mutation showed an increased sensitivity to gefitinib. Thus, we propose that gefitinib could be a potential therapeutic approach in certain colorectal cancer patients.

\section{Acknowledgements}

We would like to thank Drs A. Takayanagi, H. Tanaka, T. Sudo, T. Ohmachi, Y. Nakamura and K. Ieta for critical advice, and T. Shimooka, K. Ogata, M. Oda and M. Kayashima for technical assistance.

\section{References}

1. Huang SF, Liu HP, Li LH, et al: High frequency of epidermal growth factor receptor mutations with complex patterns in non-small cell lung cancers related to gefitinib responsiveness in Taiwan. Clin Cancer Res 10: 8195-8203, 2004.

2. Hynes NE and Lane HA: ERBB receptors and cancer: the complexity of targeted inhibitors. Nat Rev Cancer 5: 341-354, 2005.

3. Arteaga CL: Epidermal growth factor receptor dependence in human tumors: more than just expression? Oncologist 7 (Suppl 4): 31-39, 2002

4. Brabender J, Danenberg KD, Metzger R, et al: Epidermal growth factor receptor and HER2-neu mRNA expression in non-small cell lung cancer is correlated with survival. Clin Cancer Res 7: 1850-1855, 2001. 
5. Ciardiello F, Caputo R, Bianco R, et al: Inhibition of growth factor production and angiogenesis in human cancer cells by ZD1839 (Iressa), a selective epidermal growth factor receptor tyrosine kinase inhibitor. Clin Cancer Res 7: 1459-1465, 2001.

6. Grunwald V and Hidalgo M: Developing inhibitors of the epidermal growth factor receptor for cancer treatment. J Natl Cancer Inst 95: 851-867, 2003.

7. Mendelsohn J and Baselga J: Status of epidermal growth factor receptor antagonists in the biology and treatment of cancer. J Clin Oncol 21: 2787-2799, 2003.

8. Di Maio M, Gridelli C, Normanno N, Perrone F and Ciardiello F: Trying to compose the puzzle with all the pieces: epidermal growth factor receptor tyrosine kinase inhibitors in non-small cell lung cancer. J Cell Physiol 205: 355-363, 2005.

9. Fukuoka M, Yano S, Giaccone G, et al: Multi-institutional randomized phase II trial of gefitinib for previously treated patients with advanced non-small cell lung cancer (The IDEAL 1 Trial) [corrected]. J Clin Oncol 21: 2237-2246, 2003.

10. Janne PA, Gurubhagavatula S, Yeap BY, et al: Outcomes of patients with advanced non-small cell lung cancer treated with gefitinib (ZD1839, 'Iressa') on an expanded access study. Lung Cancer 44: 221-230, 2004.

11. Kris MG, Natale RB, Herbst RS, et al: Efficacy of gefitinib, an inhibitor of the epidermal growth factor receptor tyrosine kinase, in symptomatic patients with non-small cell lung cancer: a randomized trial. JAMA 290: 2149-2158, 2003.

12. Pao W, Miller V, Zakowski M, et al: EGF receptor gene mutations are common in lung cancers from 'never smokers' and are associated with sensitivity of tumors to gefitinib and erlotinib. Proc Natl Acad Sci USA 101: 13306-13311, 2004.
13. Lynch TJ, Bell DW, Sordella R, et al: Activating mutations in the epidermal growth factor receptor underlying responsiveness of non-small cell lung cancer to gefitinib. N Engl J Med 350: 2129-2139, 2004.

14. Paez JG, Janne PA, Lee JC, et al: EGFR mutations in lung cancer: correlation with clinical response to gefitinib therapy. Science 304: 1497-1500, 2004.

15. Cappuzzo F, Magrini E, Ceresoli GL, et al: Akt phosphorylation and gefitinib efficacy in patients with advanced non-small cell lung cancer. J Natl Cancer Inst 96: 1133-1141, 2004.

16. Nagahara H, Mimori K, Ohta M, et al: Somatic mutations of epidermal growth factor receptor in colorectal carcinoma. Clin Cancer Res 11: 1368-1371, 2005.

17. Livneh E, Prywes R, Kashles O, et al: Reconstitution of human epidermal growth factor receptors and its deletion mutants in cultured hamster cells. J Biol Chem 261: 12490-12497, 1986.

18. Mimori $\mathrm{K}$, Ishii $\mathrm{H}$, Nagahara $\mathrm{H}$, et al: FHIT is up-regulated by inflammatory stimuli and inhibits prostaglandin E2-mediated cancer progression. Cancer Res 66: 2683-2690, 2006.

19. Pao W, Miller VA, Politi KA, et al: Acquired resistance of lung adenocarcinomas to gefitinib or erlotinib is associated with a second mutation in the EGFR kinase domain. PLoS Med 2: e73, 2005.

20. Kobayashi S, Boggon TJ, Dayaram T, et al: EGFR mutation and resistance of non-small cell lung cancer to gefitinib. N Engl J Med 352: 786-792, 2005.

21. Greulich H, Chen TH, Feng W, et al: Oncogenic transformation by inhibitor-sensitive and -resistant EGFR mutants. PLoS Med 2: e313, 2005.

22. Kobayashi S, Ji H, Yuza Y, et al: An alternative inhibitor overcomes resistance caused by a mutation of the epidermal growth factor receptor. Cancer Res 65: 7096-7101, 2005. 